\title{
Criminologie
}

\section{Polyvictimisation et comportements à risque chez des élèves chinois du High School}

\section{Ko Ling Chan}

Volume 47, numéro 1, printemps 2014

La polyvictimisation des jeunes

URI : https://id.erudit.org/iderudit/1024008ar

DOI : https://doi.org/10.7202/1024008ar

Aller au sommaire du numéro

\section{Éditeur(s)}

Les Presses de l’Université de Montréal

ISSN

0316-0041 (imprimé)

1492-1367 (numérique)

Découvrir la revue

Citer cet article

Chan, K. L. (2014). Polyvictimisation et comportements à risque chez des élèves chinois du High School. Criminologie, 47(1), 85-103.

https://doi.org/10.7202/1024008ar
Résumé de l'article

L'étude vise à évaluer la prévalence et les facteurs associés à la polyvictimisation chez l'enfant, ainsi qu'à fournir une estimation fiable du problème dans le contexte chinois. En utilisant une procédure d'échantillonnage stratifiée à deux niveaux, 18341 élèves chinois ont été recrutés dans six villes de Chine. Ils ont été invités à remplir un questionnaire autoadministré contenant des questions sur leurs victimisations dans

l'enfance, leurs comportements de dépendance et leurs comportements sexuels à l'adolescence. La version chinoise validée du questionnaire sur la victimisation juvénile (JVQ pour l'acronyme anglophone) a été utilisée. Les taux de prévalence obtenus, portant sur l'année précédente, sont de $23,2 \%$ pour tout type de victimisation des enfants et $9,2 \%$ pour la polyvictimisation. Vivre en Chine continentale, être parmi les plus jeunes et avoir des frères et soeurs, sont les facteurs associés à l'augmentation du rapport de cote (« odds ratio ») de polyvictimisation. Certains comportements de dépendance et sexuels des adolescents, incluant les jeux de hasard, le tabagisme, l'alcoolisme, les relations sexuelles précoces, les multiples partenaires sexuels, et la grossesse chez les adolescentes, sont également significativement associés à une probabilité accrue de polyvictimisation. Les résultats de cette étude révèlent de fortes associations entre la victimisation des enfants et les comportements à risque, ce qui souligne la nécessité d'une intervention prioritaire en cas de polyvictimisation.
Ce document est protégé par la loi sur le droit d'auteur. L’utilisation des services d'Érudit (y compris la reproduction) est assujettie à sa politique d'utilisation que vous pouvez consulter en ligne.

https://apropos.erudit.org/fr/usagers/politique-dutilisation/ 


\title{
Polyvictimisation et comportements à risque chez des élèves chinois du High School ${ }^{1}$
}

\author{
Ko Ling Chan ${ }^{2,3}$ \\ Professeur associé \\ Université de Hong Kong \\ eklchan@hku.hk
}

RÉSUMÉ - L'étude vise à évaluer la prévalence et les facteurs associés à la polyvictimisation chez l'enfant, ainsi qu'à fournir une estimation fiable du problème dans le contexte chinois. En utilisant une procédure d'échantillonnage stratifiée à deux niveaux, 18341 élèves chinois ont été recrutés dans six villes de Chine. Ils ont été invités à remplir un questionnaire autoadministré contenant des questions sur leurs victimisations dans l'enfance, leurs comportements de dépendance et leurs comportements sexuels à l'adolescence. La version chinoise validée du questionnaire sur la victimisation juvénile (JVQ pour l'acronyme anglophone) a été utilisée. Les taux de prévalence obtenus, portant sur l'année précédente, sont de 23,2\% pour tout type de victimisation des enfants et 9,2\% pour la polyvictimisation. Vivre en Chine continentale, être parmi les plus jeunes et avoir des frères et sœurs, sont les facteurs associés à l'augmentation du rapport de cote ("odds ratio») de polyvictimisation. Certains comportements de dépendance et sexuels des adolescents, incluant les jeux de hasard, le tabagisme, l'alcoolisme, les relations sexuelles précoces, les multiples partenaires sexuels, et la grossesse chez les adolescentes, sont également significativement associés à une probabilité accrue de polyvictimisation. Les résultats de cette étude révèlent de fortes associations entre la victimisation des enfants et les comportements à risque, ce qui souligne la nécessité d'une intervention prioritaire en cas de polyvictimisation.

MOTS-CLÉS • Victimisation des enfants, comportements de dépendance, comportement sexuel, polyvictimisation, Chine.

1. Traduit de l'anglais par Marie Scultore.

2. Department of Social Work and Social Administration, University of Hong Kong, Pokfulam, Hong Kong, China.

3. Source de financement et reconnaissance: l'étude Optimus a été initiée et financée par la Fondation UBS Optimus. 


\section{Introduction}

Diverses études épidémiologiques ont été menées afin d'étudier la prévalence de la maltraitance des enfants dans le monde (Cawson et al., 2000; Kilpatrick et al., 2003; Trocmé et al., 2005; Chen et al., 2006, 2011). De nombreuses études se sont concentrées sur une seule forme de violence faite aux enfants, mais les enfants qui subissent une forme de victimisation sont plus à risque d'en subir d'autres formes. De plus, les effets de la victimisation multiple sont à la fois plus délétères et plus difficiles à traiter (Finkelhor et al., 2007c). Par conséquent, en se concentrant sur seulement un ou deux types de victimisation, cela peut conduire à une surestimation des impacts d'un type de victimisation. La polyvictimisation des enfants réfère à l'exposition des jeunes à de multiples formes de victimisation et à des niveaux élevés de violence, incluant les mauvais traitements physiques, la victimisation sexuelle, l'intimidation par les pairs, la criminalité conventionnelle, la violence dans le voisinage, et être témoin de violence familiale (Finkelhor et al., 2007a). Les chercheurs ont conceptualisé la polyvictimisation de l'enfant de diverses manières afin d'en étudier la prévalence. Le plus souvent, la polyvictimisation désigne les enfants ayant subi au moins quatre types de victimisation (Finkelhor et al., 2005b; Finkelhor et al., 2007a, 2007b), alors que parfois elle désigne ceux ayant subi au moins cinq types de victimisation (Finkelhor et al., 2009) ou encore ceux ayant subi des niveaux de victimisation correspondant aux $10 \%$ les plus victimisés de l'échantillon (Turner et al., 2010).

La prévalence de la polyvictimisation des enfants dans des échantillons représentatifs de la population des États-Unis varie en fonction de la définition employée. Ainsi, Finkelhor et ses collègues ont mené plusieurs études sur la polyvictimisation chez les enfants et adolescents âgés de 2 à 17 ans, et ont constaté qu'environ $18 \%$ à $22 \%$ des participants avaient été polyvictimisés dans les années précédant l'étude (Finkelhor et al., 2007b, 2009b). Dans ces études, la victimisation par les pairs et celle dans la fratrie étaient les formes les plus courantes de violence subie, alors que la victimisation sexuelle était la moins répandue. Toutefois, les jeunes victimisés sexuellement se sont révélés être le groupe le plus susceptible de faire l'expérience de multiples formes de violence, plus de $70 \%$ d'entre eux ayant déclaré avoir été victimes d'au moins quatre types de violence (Finkelhor et al., 2007b). Des prévalences similaires ont été rapportées dans les populations chinoises. Ainsi, une étude récente sur un large échantillon d'élèves du secondaire 
(12-18 ans) dans la province de Shanghai, en Chine continentale, a montré que $17 \%$ des adolescents avaient été victimes d'au moins cinq types de violence en 2012 (Dong et al., 2013). Conformément aux résultats occidentaux, la prévalence de la victimisation sexuelle $(0,4 \%)$ était la moins répandue parmi les différentes formes de violence.

\section{Facteurs de risque de victimisation des enfants et polyvictimisation}

Un nombre croissant de recherches ont été menées pour étudier les facteurs associés à la violence faite aux enfants. Les corrélats les plus fréquemment rapportés sont des facteurs démographiques tels que l'âge et le sexe de l'enfant, ainsi que le contexte familial défavorable, comme la pauvreté et les conflits conjugaux. Par exemple, Gilbert et al. (2009) ont constaté que les enfants issus de familles ayant un revenu inférieur et ceux ayant des parents moins instruits sont plus susceptibles d'être victimes de violence que leurs pairs qui ont un meilleur cadre de vie. En outre, les auteurs ont également constaté qu'il existe un modèle spécifique de victimisation selon le sexe. En effet, les filles présentent un risque plus élevé d'agressions sexuelles et de négligence, alors que les garçons sont plus à risque de punitions physiques sévères.

Outre les facteurs démographiques et familiaux, un certain nombre de facteurs psychologiques et comportementaux ont également été associés à différentes formes de violence faites aux enfants. Par exemple, les comportements délinquants et la toxicomanie ont été associés de manière significative à l'exposition à la violence familiale dans un échantillon d'adolescents taïwanais (Cheng et al., 2006). Une étude à Hong Kong chez les élèves du secondaire a également révélé une association entre la maltraitance physique et des taux plus élevés de problèmes de toxicomanie et de tabagisme (Lau et al., 2003). Les agressions sexuelles durant l'enfance sont associées aux relations sexuelles chez les adolescentes (Chen et al., 2004, 2006), aux relations sexuelles précoces, à la consommation abusive d'alcool et/ou de drogue, aux relations sexuelles non protégées et aux grossesses pendant l'adolescence (Boyer \& Fine, 1992). Dans les échantillons d'adultes, les agressions sexuelles durant l'enfance ont été associées aux relations sexuelles avec de multiples partenaires (Widom \& Kuhns, 1996).

La littérature sur les corrélats de la polyvictimisation est plus rare. Parmi le peu d'études existantes, celles de Finkelhor et son équipe ont 
révélé que des taux de prévalence plus élevés de polyvictimisation chez les enfants âgés de 2 à 17 ans au cours d'une année étaient associés aux adolescents les plus âgés, au sexe masculin, au chômage des parents, aux conflits parentaux, aux difficultés financières familiales et à la toxicomanie des jeunes (Finkelhor et al., 2007a, 2007b, 2009a; Turner et al., 2010). Faisant écho à ces conclusions, une étude américaine a rapporté une association entre polyvictimisation, alcoolisme, et toxicomanie, auprès d'un échantillon national de plus de 3000 adolescents (Ford et al., 2010).

\section{La présente étude}

Dans cette étude, nous nous intéressons à la prévalence de la polyvictimisation dans un échantillon large et diversifié, obtenu dans six villes chinoises. L'objectif est de fournir un portrait systématique et fiable de la victimisation des enfants en Chine à l'aide du questionnaire sur la victimisation juvénile (Juvenile Victimization Questionaire [JVQ]); voir Finkelhor et al., 2005a) qui a été utilisé dans les études occidentales. Compte tenu de l'association entre la victimisation des enfants et des adolescents et des comportements à risque tels que les comportements de dépendance et les activités sexuelles non protégées, il est possible que les polyvictimes soient plus susceptibles d'adopter ces comportements. Notre deuxième objectif est donc d'examiner l'association entre la victimisation, les comportements de dépendance et les activités sexuelles à risque des adolescents. Conformément à la littérature concernant une forme unique de victimisation, les comportements à risque des adolescents devraient être associés à une plus forte probabilité de polyvictimisation.

\section{Méthodes}

\section{Les participants}

Considérant l'énorme population et les diverses communautés chinoises, il est très difficile de recruter un échantillon réellement représentatif de l'ensemble du pays. Une stratégie plus pratique a été adoptée pour cette étude, en sélectionnant des villes de différentes régions géographiques en Chine, incluant Tianjin, Shenzhen, Shanghai, Xi'an, Wuhan et Hong Kong. Ces cinq villes sont situées dans le nord, le sud, l'est, l'ouest et les régions centrales de la Chine et sont généralement considérées comme ayant des caractéristiques socioéconomiques, politiques et 
juridiques qui diffèrent de celles de Hong Kong, l'ancienne colonie britannique, devenue chinoise en 1997.

Dans cette étude, une stratégie d'échantillonnage stratifié à deux niveaux a été employée afin de recruter des élèves du secondaire de novembre 2009 à juillet 2010. Un total de 18341 adolescents âgés de 15 à 17 ans (53,3\% de garçons, âge moyen de 15,9 ans [SD = 0,1]) a été recruté. Environ $21,4 \%$ des élèves étaient originaires de Hong Kong, et les $78,6 \%$ restants vivaient dans les cinq villes du continent. D'autres informations concernant la méthodologie, la procédure et les données démographiques des participants de cette étude sont disponibles dans d'autres publications (Chan et al., 2012; Chan, 2013).

\section{Mesures}

\section{Victimisation des enfants}

La victimisation des enfants fut mesurée à l'aide de la version chinoise $\mathrm{du}$ questionnaire sur la victimisation juvénile (JVQ; Finkelhor et al., 2005a; Chan et al., 2011), une échelle de 34 items portant sur cinq aspects de la victimisation et de la violence vécues par les enfants et les adolescents. Ces cinq aspects incluent (a) la criminalité conventionnelle (8 items), (b) la maltraitance des enfants (4 items), (c) la victimisation de la part des pairs et de la fratrie (6 items), (d) la victimisation sexuelle (7 items), et (e) être témoin de victimisation de manière directe ou indirecte (9 items). Cette version chinoise fut traduite en utilisant une procédure de traduction inversée (Chan et al., 2011). L'étude documente la victimisation vécue (a) au cours de l'année précédant l'étude et (b) au cours de la vie. Une échelle de réponse en 3 points a été utilisée, où 1 indique avoir subi ou avoir été témoin d'un type spécifique de violence dans l'année précédente, 2 indiquant avoir subi ou avoir été témoin d'un type spécifique de violence avant l'année précédente, et 0 signifiant n'avoir jamais subi, ou n'avoir jamais été témoin de violence. Les participants ayant répondu 1 à n'importe quel élément ont été inclus dans la prévalence annuelle, et ceux ayant répondu 1 ou 2 à n'importe quel élément ont été inclus dans la prévalence à vie. Les répondants qui ont déclaré au moins quatre types de victimisation étaient considérés comme étant polyvictimisés (Finkelhor et al., 2005b). Le JVQ chinois a démontré une fiabilité modérée à excellente avec des valeurs alpha de Cronbach variant de ,63 (maltraitance) à ,97 (victimisation sexuelle) pour l'ensemble des cinq modules, et de, 90 pour l'échelle globale. 


\section{Les comportements de dépendance}

Quatre items ont été développés afin d'évaluer si les élèves avaient eu des comportements de dépendance au cours de leur vie. Les questions portaient sur quatre types de comportement de dépendance, y compris (1) le jeu fréquent, (2) fumer fréquemment, (3) l'abus d'alcool, et (4) la toxicomanie. Les articles étaient tous notés sur une échelle dichotomique «oui» «non».

\section{Comportements sexuels des adolescents}

Quatre items ont été employés afin de capturer les comportements sexuels des adolescents au cours de leur vie. Ces items incluent (1) avoir déjà eu ou non des relations sexuelles, (2) l'âge des premières relations sexuelles, (3) le nombre de partenaires sexuels depuis les premières relations sexuelles, et (4) le nombre de grossesses chez les adolescentes ainsi que l'âge lors de la grossesse. Les participants devaient répondre par des nombres précis.

\section{Caractéristiques démographiques}

Elles comprennent l'âge, le sexe, le nombre de frères et sœurs, et la ville dans laquelle l'école des participants était située.

\section{Les analyses statistiques}

La prévalence de la victimisation, de la polyvictimisation, des comportements de dépendance et des comportements sexuels des adolescents a été mesurée. Pour examiner les associations entre la victimisation des enfants, les caractéristiques démographiques, les comportements de dépendance et les comportements sexuels des adolescents, une analyse de régression logistique structurelle en deux phases a été réalisée. L'hypothèse soutenant l'analyse était que les variables indépendantes de la première phase affecteraient ceux de la phase subséquente, mais pas l'inverse. Dans la phase 1 , une régression logistique a été réalisée avec la victimisation (la variable dépendante) et chacun des facteurs démographiques et des comportements de dépendance, avec un ajustement des autres facteurs inclus dans l'équation. Aucun problème de multicolinéarité entre les variables n'a été décelé. Dans la phase 2 , chacun des comportements sexuels des adolescents a été inclus individuellement dans la régression de victimisation en contrôlant tous les 
autres facteurs, soit les variables démographiques, les comportements de dépendance et les autres comportements sexuels. Dans toutes les analyses, les données manquantes furent traitées par une suppression par paires. La qualité d'ajustement des modèles de régression a été testée avec le test de Hosmer et Leshow (HL). Une valeur de $p$ inférieure à 0,05 a été considérée comme statistiquement significative, et toutes les analyses statistiques ont été réalisées en utilisant SPSS version 17.

\section{Résultats}

Importance de la victimisation des enfants, polyvictimisation et comportements à risque des adolescents

Le tableau 1 montre la prévalence de la victimisation des enfants selon le nombre de victimisations vécues. Environ $24,2 \%$ et $23,2 \%$ des élèves ont subi au moins un type de victimisation au cours de leur vie ou de l'année précédente. Ce sont $14,0 \%$ et $9,2 \%$ des élèves qui ont été polyvictimisés (au moins quatre types de victimisation) dans leur vie ou au cours de l'année précédente, respectivement. Concernant les différences entre les sexes, les garçons (10,6\%-15,8\%) étaient plus susceptibles que les filles (7,5\%-12,0\%) d'être des polyvictimes.

Les prévalences à vie et annuelle des différents types de victimisation variaient respectivement de $8,0 \%$ à $57,1 \%$ et de $6,4 \%$ à $43,1 \%$. Les résultats détaillés des prévalences de chaque type de victimisation spécifique ainsi que leurs différences selon le genre sont disponibles dans une autre publication (Chan et al., 2012).

Le tableau 2 présente la prévalence des comportements de dépendance et des comportements sexuels des élèves. La forme la plus répandue de comportements de dépendance était le jeu, suivie de la consommation abusive d'alcool et du tabagisme. Le comportement le moins fréquent était la toxicomanie. En ce qui concerne les comportements sexuels des adolescents, environ 3,6\% de l'échantillon avait eu des relations sexuelles. Plus de $2 \%$ des adolescents avaient vécu cette expérience avant d'avoir 16 ans, et $0,1 \%$ ont eu une grossesse adolescente. Les différences entre les sexes ont révélé que significativement plus de garçons que de filles présentaient des comportements de dépendance et des comportements sexuels à risque $(p<, 05)$. 
TABLEAU 1

Prévalences à vie et annuelle de la victimisation et de la polyvictimisation des adolescents en fonction du sexe $(n=18341)$

\begin{tabular}{|c|c|c|c|c|c|c|c|}
\hline \multirow[t]{2}{*}{$\mathrm{Nb}$ de types de victimisation } & \multicolumn{3}{|c|}{ Prévalence à vie } & \multicolumn{4}{|c|}{ Prévalence annuelle } \\
\hline & $\begin{array}{c}\text { Tous } \\
(n=18341) \\
\%\end{array}$ & $\begin{array}{c}\text { Garçons } \\
(n=9773) \\
\%\end{array}$ & $\begin{array}{c}p \text {-value }^{\text {a }} \\
<, 001\end{array}$ & $\begin{array}{c}\text { Tous } \\
(n=18341) \\
\%\end{array}$ & $\begin{array}{c}\text { Filles } \\
(n=8568) \\
\%\end{array}$ & $\begin{array}{c}\text { Garçons } \\
(n=9773) \\
\%\end{array}$ & $\begin{array}{c}p \text {-value } \\
<, 001\end{array}$ \\
\hline 1 type & 24,2 & 23,3 & & 23,2 & 24,7 & 21,9 & \\
\hline 2 types & 18,6 & 17,4 & & 16,3 & 16,9 & 15,7 & \\
\hline 3 types & 14,9 & 15,5 & & 11,3 & 10,4 & 12,2 & \\
\hline 4 types et plus (poly-victimisation) & 14,0 & 15,8 & & 9,2 & 7,5 & 10,6 & \\
\hline
\end{tabular}

a $p$-value du $\chi^{2}$ 
TABLEAU 2

Prévalence à vie des comportements de dépendance et des comportements sexuels chez les adolescents selon le sexe $(n=18$ 341)

\begin{tabular}{|c|c|c|c|c|}
\hline \multirow[b]{2}{*}{ Comportements } & \multicolumn{4}{|c|}{ Prévalence à vie } \\
\hline & $\begin{array}{c}\text { Tous } \\
\left(n=\begin{array}{c}18 \\
\% \\
\%\end{array}\right.\end{array}$ & $\begin{array}{c}\text { Filles } \\
(n=8568) \\
\%\end{array}$ & $\begin{array}{c}\text { Garçons } \\
\left(\begin{array}{c}n=9 \\
973) \\
\%\end{array}\right.\end{array}$ & $p$-value ${ }^{a}$ \\
\hline \multicolumn{5}{|c|}{ Comportements de dépendance } \\
\hline Jeux & 6,1 & 3,4 & 8,4 & $<, 001$ \\
\hline Tabagisme & 4,5 & 1,7 & 6,9 & $<, 001$ \\
\hline Alcoolisme & 5,4 & 3,2 & 7,3 & $<, 001$ \\
\hline Toxicomanie & 0,9 & 0,7 & 1,1 &, 006 \\
\hline \multicolumn{4}{|c|}{ Expérience de rapports sexuels } & $<, 001$ \\
\hline Oui & 3,6 & 2,2 & 4,8 & \\
\hline Non & 92,6 & 94,1 & 91,3 & \\
\hline Valeur manquante & 3,8 & 3,7 & 3,9 & \\
\hline \multicolumn{5}{|l|}{ Comportements sexuels } \\
\hline \multicolumn{4}{|c|}{ Âge à la première expérience } & 007 \\
\hline$<16$ ans & 2,1 & 1,4 & 2,7 & \\
\hline$>16$ ans & 1,4 & 0,7 & 2,0 & \\
\hline Valeurs manquantes & 3,9 & 3,8 & 4,0 & \\
\hline Non applicables ${ }^{b}$ & 92,6 & 94,1 & 91,3 & \\
\hline \multicolumn{4}{|l|}{ Partenaires sexuels } & 013 \\
\hline 1 & 0,9 & 0,4 & 1,1 & \\
\hline$>2$ & 1,8 & 1,1 & 2,5 & \\
\hline Valeurs manquantes & 4,7 & 4,4 & 5,1 & \\
\hline Non applicable ${ }^{b}$ & 92,6 & 94,1 & 91,3 & \\
\hline \multicolumn{4}{|l|}{ Grossesse précoce } & $<, 001$ \\
\hline 1 & 0,1 & 0,1 & $0,2^{\mathrm{c}}$ & \\
\hline$\geq 2$ & 0,5 & 0,2 & $0,7^{c}$ & \\
\hline 0 & 3,0 & 1,9 & 3,9 & \\
\hline Non applicableb & 92,6 & 94,1 & 91,3 & \\
\hline
\end{tabular}

a $p$-value du $\chi^{2}$.

b Non applicable = participants sans expérience sexuelle.

Grossesse de la partenaire sexuelle. 
TABLEA U 3

Classification des adolescents selon les caractéristiques démographiques, les comportements de dépendance et les comportements sexuels en fonction du nombre de victimisations au cours de l'année précédente $(n=18341)$

\begin{tabular}{|c|c|c|c|c|}
\hline & \multicolumn{3}{|c|}{ Pourcentage de victimes (année précédente) } & \multirow[t]{2}{*}{$p$-value } \\
\hline Variable & $\begin{array}{c}\text { Absence de } \\
\text { victimisation } \\
\%\end{array}$ & $\begin{array}{c}1 \text { à } 3 \text { types de } \\
\text { victimisation } \\
\%\end{array}$ & $\begin{array}{c}\text { Poly- } \\
\text { victimisation } \\
\% \\
\end{array}$ & \\
\hline \multicolumn{5}{|c|}{ Caractéristiques démographiques } \\
\hline Lieux de recherche & & & & $<, 001$ \\
\hline Continent chinois & 37,7 & 52,3 & 10,1 & \\
\hline Hong Kong & 48,8 & 45,3 & 5,9 & \\
\hline Genre & & & & $<, 001$ \\
\hline Garçon & 39,6 & 49,8 & 10,6 & \\
\hline Fille & 40,5 & 51,9 & 7,5 & \\
\hline$\hat{A} g e$ & 15,9 & 15,9 & 15,7 & $<, 001^{\mathrm{b}}$ \\
\hline Enfant unique & & & & $<, 001$ \\
\hline Oui & 48,4 & 44,2 & 7,4 & \\
\hline Non & 34,8 & 55,1 & 10,2 & \\
\hline \multicolumn{5}{|c|}{ Comportements de dépendance } \\
\hline Jeux & 29,0 & 56,7 & 14,3 & $<, 001$ \\
\hline Tabagisme & 19,0 & 56,2 & 24,8 & $<, 001$ \\
\hline Alcoolisme & 24,5 & 56,5 & 19,1 & $<, 001$ \\
\hline Toxicomanie & 35,7 & 43,1 & 21,2 & $<, 001$ \\
\hline \multicolumn{5}{|c|}{ Comportements sexuels } \\
\hline \multicolumn{4}{|c|}{ Âge lors de la première relation sexuelle } & $<, 001^{\mathrm{c}}$ \\
\hline$<16$ ans & 15,3 & 49,6 & 35,1 & \\
\hline$\geq 16$ ans & 18,6 & 55,7 & 25,7 & \\
\hline $\begin{array}{l}\text { Aucune relation } \\
\text { sexuelle }\end{array}$ & 41,2 & 50,5 & 8,2 & \\
\hline Partenaires sexuels & & & & $<, 001^{\mathrm{c}}$ \\
\hline 1 & 17,8 & 55,4 & 26,7 & \\
\hline$>2$ & 16,3 & 51,9 & 31,8 & \\
\hline $\begin{array}{l}\text { Aucun partenaire } \\
\text { sexuel }\end{array}$ & 41,2 & 50,5 & 8,2 & \\
\hline Grossesse précoce & & & & $<, 001^{\mathrm{c}}$ \\
\hline 1 & 17,7 & 57,1 & 25,3 & \\
\hline$>2$ & 14,9 & 45,6 & 39,5 & \\
\hline $\begin{array}{l}\text { Aucune grossesse } \\
\text { précoce }\end{array}$ & 41,2 & 50,5 & 8,3 & \\
\hline
\end{tabular}

$p$-value du $\chi^{2}$.

b $p$-value du T-test.

$p$-value d'ANOVA. 
TABLEA U 4

Associations indépendantes entre les caractéristiques démographiques, les comportements de dépendance, les comportements sexuels et la polyvictimisation durant l'année précédant l'étude

\begin{tabular}{|c|c|c|}
\hline Variable & $\begin{array}{c}\text { Rapport } \\
\text { de cote } \\
\text { (odds ratio) }\end{array}$ & $\begin{array}{l}\text { (95\% Intervalle } \\
\text { de confiance) }\end{array}$ \\
\hline Phase $1^{\text {a }}$ & $(n=15283)$ & $R^{2 b}=8,6 \%, p^{c}<0,001$ \\
\hline \multicolumn{3}{|l|}{ Caractéristiques démographiques } \\
\hline \multicolumn{3}{|l|}{ Lieux de recherche } \\
\hline 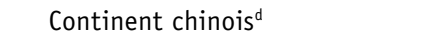 & $1,95^{* * *}$ & $(1,80,2,12)$ \\
\hline \multicolumn{3}{|l|}{ Genre } \\
\hline Garçon & 1,00 & $(0,94,1,07)$ \\
\hline$\hat{A} g e$ & $0,91^{* * *}$ & $(0,88,0,94)$ \\
\hline Membre d'une fratrie & $1,89 * * *$ & $(1,76,2,01)$ \\
\hline \multicolumn{3}{|l|}{ Comportements de dépendance ${ }^{e}$} \\
\hline Jeux de hasard & $1,40^{* * *}$ & $(1,26,1,55)$ \\
\hline Tabagisme & $2,37^{* * *}$ & $(2,07,2,72)$ \\
\hline Consommation excessive d'alcool & $1,60 * * *$ & $(1,43,1,80)$ \\
\hline Toxicomanie & 0,78 & $(0,58,1,05)$ \\
\hline \multicolumn{3}{|l|}{ Phase 2} \\
\hline \multicolumn{3}{|c|}{ Âge lors de la première expérience sexuelle } \\
\hline$<16$ ans & $3,79 * * *$ & $(3,02,4,78)$ \\
\hline$\geq 16$ ans & $2,92 * * *$ & $(2,20,3,86)$ \\
\hline \multicolumn{3}{|l|}{ Nombre de partenaires sexuels } \\
\hline 1 & $2,52 * * *$ & $(1,63,3,89)$ \\
\hline$>22$ & $3,53^{* * *}$ & $(2,88,4,32)$ \\
\hline \multicolumn{3}{|l|}{ Grossesse précoce } \\
\hline Oui & $3,83^{* * *}$ & $(2,52,5,84)$ \\
\hline
\end{tabular}

${ }^{*} p<, 05 . ;{ }^{* *} p<, 01 . ;{ }^{* * *} p<, 001$.

a Les variables de la phase 1 ont été ajustées aux autres variables dans la même phase, et les variables de la phase 2 ont été ajustées à toutes les variables de la phase 1 ainsi qu'aux variables de la même phase.

b Le $R^{2}$ ajusté de Nagelkerke a été utilisé.

c $P$-value par l'essai du rapport de vraisemblance (likelihood ratio test).

d Continent chinois = tous les lieux en Chine continentale, à savoir e Shanghai, Shenzhen, Tianjin,

Wuhan et Xi'an. Groupe référent $=$ Hong Kong.

e Groupe référent = aucune expérience similaire. 
Facteurs associés à la victimisation et à la polyvictimisation

Le tableau 3 montre la répartition des élèves selon leurs caractéristiques démographiques ainsi que les comportements de dépendance et sexuels en fonction du nombre d'incidents de victimisation vécus durant l'année précédente. Les résultats montrent que la répartition des élèves selon les facteurs étudiés diffère significativement entre les non-victimes, les jeunes victimes d'incidents de victimisation (1 à 3 ) et les polyvictimes (tous les $p<, 001$ ).

Le tableau 4 présente les résultats de l'analyse de régression logistique à deux phases. En ce qui concerne les caractéristiques démographiques, le fait de vivre dans les villes du continent de la Chine et d'avoir de la fratrie était associé à une augmentation du rapport de cote (odds ratios) de polyvictimisation, par rapport aux groupes de référence, alors qu'être plus âgé était lié à une diminution des rapports de cote de la polyvictimisation pendant l'année précédente $(p<, 001)$.

Les jeux de hasard fréquents, le tabagisme excessif et la consommation abusive d'alcool sont trois types de comportement de dépendance associés à une augmentation des risques de polyvictimisation $(p<, 001)$. Cependant, l'association entre la polyvictimisation et la toxicomanie n'était pas significative.

En ce qui concerne les comportements sexuels, avoir eu des rapports sexuels, quel que soit l'âge de la première relation, était associé à une augmentation des risques de polyvictimisation. De plus, les partenaires sexuels multiples et les grossesses précoces étaient aussi associés à une augmentation des rapports de cote de la polyvictimisation $(p<, 001)$.

\section{Discussion}

En utilisant un échantillon large et diversifié recruté dans différentes zones géographiques en Chine, cette étude a fourni des estimations plus fiables de la prévalence de la polyvictimisation, des comportements de dépendance et des activités sexuelles chez les étudiants chinois de l'école secondaire. La prévalence de tout type de violence envers les enfants révélée par cette étude était faible (24,2\% pour la vie et $23,2 \%$ pour l'année précédente) par rapport à celle des États-Unis où des études épidémiologiques sur la victimisation ont révélé qu'environ $66 \%$ (Turner et al., 2010) à $71 \%$ (Finkelhor et al., 2005a) des enfants étaient victimes d'un ou de plusieurs types de victimisation. Le résultat est également inférieur aux chiffres obtenus dans une étude auprès d'élèves 
du secondaire d'une ville du continent chinois qui indique une prévalence de $69 \%$ (Cheng et al., 2010). On remarque une différence similaire concernant la polyvictimisation durant l'année précédente, qui étaient de 9,2\% dans cette étude, de $18 \%$ (Finkelhor et al., 2007b) à $22 \%$ (Finkelhor et al., 2005b) dans les études américaines, et de $17 \%$ (Cheng et al., 2010) dans l'étude chinoise continentale.

Il y avait des différences significatives selon le sexe quant à la prévalence de la polyvictimisation, les garçons étant plus susceptibles que les filles de déclarer avoir été polyvictimes. Cette tendance à prédominance masculine est conforme aux conclusions d'études antérieures, qui démontrent que les garçons sont souvent soumis à plusieurs types de victimisation tels l'agression, l'intimidation et les crimes contre la propriété (Finkelhor et al., 2007a, 2009; Finkelhor et al., 2009a; Wang et al., 2010). Tel que proposé dans un récent article (Chan et al., 2012), deux mécanismes pourraient expliquer la prédominance masculine. Premièrement, elle pourrait être le reflet de la sous-déclaration d'une expérience violente par les filles chinoises. Deuxièmement, il est possible qu'il y ait une différence réelle de prévalence de victimisation entre les garçons et les filles en Chine, car le contrôle parental y est généralement plus strict sur les filles que sur les garçons. Ainsi, les filles, qui sont censées rester à la maison plutôt que de sortir fréquemment, seraient moins susceptibles d'être victimes de violence extra-familiale et d'être témoins de violence dans la communauté.

Nous avons trouvé certains facteurs associés à la polyvictimisation. L'emplacement de l'école était un facteur significatif. Comparativement aux étudiants de Hong Kong, les étudiants en provenance de la Chine continentale ont une plus forte probabilité d'être l'objet de divers types de victimisation. Compte tenu de la nature transversale de cette étude, les données ne permettent pas de déterminer quels mécanismes peuvent expliquer ce phénomène. Cependant, cela pourrait refléter des différences entre les niveaux de supervision parentale en Chine et à Hong Kong. Contrairement aux études précédentes soulignant que les enfants plus âgés étaient plus à risque d'être à la fois victimes et polyvictimes (Finkelhor et al., 2005a, 2005b, 2007a; Turner et al., 2010), l'âge est négativement associé à la victimisation dans la présente étude. Cela pourrait être en lien avec la faible variation d'âge de notre échantillon (15-17 ans), qui est relativement plus âgé par rapport aux études précédentes. Les adolescents dans cette étude avaient possiblement de meilleures capacités d'autoprotection, puisqu'ils approchaient de l'âge 
adulte. Nous avons également constaté qu'avoir de la fratrie augmentait la probabilité d'être victimisé. En vertu de la politique de l'enfant unique, les familles chinoises avec un seul enfant ont concentré leur attention et leurs ressources sur ce dernier afin de lui permettre d'avoir des avantages par rapport à ses pairs (Ma et al., 2007). Ces parents sont peu propices à abuser eux-mêmes de l'enfant ou à le laisser se faire victimiser dans un autre environnement. Dans le même ordre d'idées, les parents de plusieurs enfants peuvent avoir eu à diviser leurs ressources et ainsi limiter l'attention et/ou leur capacité à protéger chaque enfant. La politique de l'enfant unique en Chine fournit également plus de ressources - et des avantages (allocations) - aux parents d'enfant unique, alors que ce système met une pression supplémentaire sur les ressources de la famille et punit financièrement ceux qui ont plus d'un enfant (par exemple: des amendes ou des restrictions à l'emploi). Ces facteurs pourraient être susceptibles d'aider à protéger l'enfant unique de la victimisation.

Appuyant notre hypothèse, les adolescents qui s'étaient engagés dans des comportements sexuels ou de dépendance présentaient des risques significativement plus élevés d'être victimisés. Tous les types de comportement de dépendance inclus dans cette étude étaient associés à un risque accru de victimisation, ce qui correspond à la littérature existante traitant de l'association entre les comportements à risque et la victimisation (Lau et al., 2003; Cheng et al., 2006). Un mécanisme explicatif possible est que les enfants issus de milieux et de communautés défavorisés, tels que les quartiers pauvres et les écoles disposant de ressources limitées, sont plus susceptibles de développer des comportements délinquants et risqués et d'être impliqués dans la criminalité et la violence en raison de la moindre supervision parentale et scolaire (Finkelhor et al., 2009).

Les résultats soulignent que les comportements sexuels des adolescents sont un facteur important significativement associé à la victimisation. Dans cette étude, les étudiants qui ont eu des rapports sexuels avant 16 ans, de multiples partenaires sexuels ou des grossesses durant l'adolescence étaient beaucoup plus susceptibles d'avoir été victimisés. Bien que la cause de cette relation ne soit pas encore claire, de nombreuses études ont trouvé des associations similaires. Par exemple, l'agression sexuelle dans l'enfance a été associée aux rapports sexuels précoces chez des étudiantes de l'école secondaire (Chen et al., 2004, 2006), à un risque accru d'avoir des relations sexuelles à un jeune âge, 
à l'abus d'alcool et de drogues, aux relations sexuelles non protégées et aux grossesses à l'adolescence (Boyer \& Fine, 1992). De plus, la maltraitance a été associée aux comportements sexuels des élèves de l'école primaire (Lau et al., 2005 ; Teixeria \& Taquette, 2010). Une explication possible serait que l'expérience de victimisation précoce augmenterait la tendance des enfants à être impliqués dans des relations sexuelles, ce qui les placerait ensuite à risque d'être la cible d'autres victimisations. Un résultat intrigant de cette étude est le fait que les adolescents ayant eu leur première relation sexuelle avec un partenaire du même sexe avaient des taux plus élevés de polyvictimisation. Puisquaucune étude n'a encore examiné ce facteur, nous ne pouvons conclure qu'il s'agit d'un modèle universel. Cependant, il est possible que les jeunes homosexuels puissent être plus à risque d'être victime parce qu'ils appartiennent à une minorité. D'autres études sont nécessaires afin de vérifier cette hypothèse.

\section{Les limites de cette étude}

À notre connaissance, cette étude est la première à fournir des preuves solides de l'association entre les comportements à risque et la victimisation des enfants dans la population chinoise. Toutefois, plusieurs limites doivent être considérées. Tout d'abord, l'échantillon a été limité à six villes de Chine. Comme la Chine est un grand pays, avec un développement inégal dans diverses régions, les résultats de cette étude ne peuvent être généralisés à toute la nation. En outre, en raison de la nature des données transversales utilisées, il n'est pas possible d'établir de causalité entre les facteurs. Une étude longitudinale sera nécessaire afin d'établir la direction de la relation entre les variables. Par ailleurs, l'étude actuelle est axée uniquement sur les associations entre les comportements à risque et la victimisation. D'autres facteurs sont associés à la victimisation des enfants, tels que les caractéristiques de la personnalité, ainsi que la violence familiale et/ou la violence communautaire. Il n'a donc pas été possible dans cette étude d'examiner les interactions entre ces facteurs de manière à fournir une image complète de la victimisation des enfants. De plus, l'échantillon n'inclut pas les jeunes de moins de 15 ans, limitant la portée de l'étude. Bien que les résultats soient plus spécifiques et applicables à la population cible, la généralisation des conclusions se limite aux jeunes plus âgés. 
Malgré les limites, cette étude est parmi les premières à utiliser un échantillon large et diversifié en Chine, et fournit ainsi certaines statistiques vitales concernant la victimisation et les comportements à risque chez les enfants de ce pays. De plus, les informations proviennent des adolescents eux-mêmes pour cette étude, ce qui rend les résultats plus fiables que si l'information avait été obtenue à travers les parents, qui ont tendance à être plus biaisés par désirabilité sociale.

\section{Conclusion}

Les conclusions de l'étude ont des implications dans le domaine de la santé publique. Cette étude met en évidence la nécessité d'une intervention ou d'un traitement prioritaire en cas de polyvictimisation. Puisque les enfants qui subissent de multiples victimisations sont susceptibles de subir des conséquences plus graves et moins réversibles (Finkelhor et al., 2007c) et présentent une plus grande probabilité à s'impliquer dans divers comportements à risque, ils ont besoin d'une intervention rapide afin de prévenir ces conséquences négatives. Cette étude souligne également la nécessité d'un traitement plus englobant qui intègre les comportements à risque et la victimisation et aborde ces deux problèmes à la fois. En raison de l'interdépendance de ces deux phénomènes, des interventions qui les prennent en considération simultanément seraient mieux placées pour aider l'adaptation et le développement optimal des enfants.

\section{Références}

Boyer, D., \& Fine, D. (1992). Sexual abuse as a factor in adolescent pregnancy and child maltreatment. Family Planning Perspectives, 24 (1), 4-19.

Cawson, P., Wattam, C., Brooker, S., \& Kelly, G. (2000). Child maltreatment in the United Kingdom: A study of the prevalence of abuse and neglect. London: NSPCC.

Chan, K. L. (2013). Victimization and Poly-Victimization Among School-Aged Chinese Adolescents: Prevalence and Associations with Health. Preventive Medicine, 56 (3-4), 207-210.

Chan, K. L., Yan, E., Brownridge, D. A. \& Ip, P. (2012). Associating Child Sexual Abuse with Child Victimization in China. The Journal of Pediatrics, 162 (5), 1028-1034.

Chan, K. L., Brownridge, D. A., Yan, E., Fong, D. Y. T., \& Tiwari, A. (2011). Child maltreatment polyvictimization: Rates and short-term effects on adjustment in a representative Hong Kong sample. Psychology of Violence, 1 (1), 4-15. 
Chan, K. L., Fong, D. Y. T., Yan, E., Chow, C. B., \& Ip, P. (2011). Validation of the Chinese Juvenile Victimization Questionnaire. Hong Kong Journal of Paediatrics, 16 (1), 17-24.

Chen, J., Dunne, M. P., \& Han, P. (2004). Child Sexual Abuse in China: A Study of Adolescents in Four Provinces. Child Abuse $\mathfrak{F}$ Neglect, 28 (11), 1171-1186.

Chen, J., Dunne, M. P., \& Han, P. (2006). Child sexual abuse in Henan province, China: Associations with sadness, suicidality, and risk behaviors among adolescent girls. Journal of Adolescent Health, 38 (5), 544-549.

Cheng, C. P., Yen, C. F., Hsu, C. C., Liu, S. C., Huang, C. F., Ko, C. H., \& Yen, J. Y. (2006). Relationships among mental health status, social context, and demographic characteristics in Taiwanese aboriginal adolescents: A structural equation model. Psychiatry and Clinical Neurosciences, 60 (5), 575-583.

Cheng, P. X., Cao, F. L., Liu, J. J., \& Chen, Q. Q. (2010). Reliability and validity of Chinese self-report version of Juvenile Victimization Questionnaire in middle school students. Chinese Journal of Clinical Psychology, 18 (4), 460-462.

Dong, F., Cao, F., Cheng, P., Cui, N., \& Li, Y. (2013). Prevalence and associated factors of poly-victimization in Chinese adolescents. Scandinavian Journal of Psycology, 54 (5), 415-422. [Epub ahead of print]. doi : 10.1111/ 2jop.12059.

Finkelhor, D., Hamby, S. L., Ormrod, R. K., \& Turner, H. A. (2005). The Juvenile Victimization Questionnaire: reliability, validity, and national norms. Child Abuse Er Neglect, 29 (4), 383-412.

Finkelhor, D., Ormrod, R. K., Turner, H. A., \& Hamby, S. L. (2005a). The victimization of children and youth: A comprehensive, national survey. Child Maltreatment, 10 (1), 5-25.

Finkelhor, D., Ormrod, R. K., Turner, H. A., \& Hamby, S. L. (2005b). Measuring poly-victimization using the Juvenile Victimization Questionnaire. Child Abuse $\mathcal{*}$ Neglect, 29 (11), 1297-1312.

Finkelhor, D., Ormrod, R. K., \& Turner, H. A. (2007a). Poly-victimization: A neglected component in child victimization. Child Abuse $\mathfrak{F}$ Neglect, 31 (1), 7-26.

Finkelhor, D., Ormrod, R. K., \& Turner, H. A. (2007b). Polyvictimization and trauma in a national longitudinal cohort. Development and Psychopathology, 19 (1), 149-166.

Finkelhor, D., Ormrod, R. K., \& Turner, H. A. (2007c). Re-victimization patterns in a national longitudinal sample of children and youth. Child Abuse $\sim$ Neglect, 31 (5), 479-502.

Finkelhor, D., Ormrod, R. K., Turner, H. A., \& Holt, M. (2009). Pathways to poly-victimization. Child Maltreatment, 14 (4), 316-329.

Finkelhor, D., Ormrod, R. K., \& Turner, H. A. (2009a). The developmental epidemiology of childhood victimization. Journal of interpersonal violence, 24 (5), 711-731.

Finkelhor, D., Ormrod, R. K., \& Turner, H. A. (2009b). Lifetime assessment of poly-victimization in a national sample of children and youth. Child Abuse $\mathfrak{O}$ Neglect, 33 (7), 403-411. 
Ford, J. D., Elhai, J. D., Connor, D. F., \& Frueh, B. C. (2010). Poly-victimization and risk of posttraumatic, depressive, and substance use disorders and involvement in delinquency in a national sample of adolescents. Journal of Adolescent Health, 46 (6), 545-552.

Gilbert, R., Widom, C. S., Browne, K., Fergusson, D., Webb, E., \& Janson, S. (2009). Burden and consequences of child maltreatment in high-income countries. The Lancet, 373 (9657), 68-81.

Kilpatrick, D. G., Saunders, B. E., \& Smith, D. W. (2003). Youth victimization: Prevalence and implications. Research in brief. Washington, DC: Office of Justice Programs, National Institute of Justice.

Lau, J. T. F., Chan, K. K., Lam, P. K. W., Choi, P. Y. W., \& Lai, K. Y. C. (2003). Psychological correlates of physical abuse in Hong Kong Chinese adolescents. Child Abuse of Neglect, 27 (1), 63-75.

Lau, J. T. F., Kim, J. H., Tsui, H.-Y., Cheung, A., Lau, M., \& Yu, A. (2005). The relationship between physical maltreatment and substance use among adolescents : a survey of 95,788 adolescents in Hong Kong. Journal of Adolescent Health, 37 (2), 110-119.

Ma, H., Liu, E., Liu, X., \& Liu, F. (2007). Family and marriage in China after the implementation of the single-child policy. In A. S. Loveless \& T. B. Holman (eds.), The family in the new millennium: World voices supporting the "natural" clan. Westport, CT: Greenwood Publishing Group, Inc.

Teixeira, S. A. M., \& Taquette, S. R. (2010). Violence and unsafe sexual practices in adolescents under 15 years of age. Revista Da Associacao Medica Brasileira, 56 (4), 440-446.

Trocmé, N., Fallon, B., MacLaurin, B., Daciuk, J., Felstiner, C., Black, T., \& Turcotte, D. (2005). Canadian incidence study of reported child abuse and neglect: Major findings - 2003. Ottawa, Ontario, Canada: Minister of Public Works and Government Services Canada.

Turner, H. A., Finkelhor, D., \& Ormrod, R. (2010). Poly-victimization in a national sample of children and youth. American Journal of Preventive Medicine, 38 (3), 323-330.

Wang, Z., Du, J., Sun, H., Wu, H., Xiao, Z., \& Zhao, M. (2010). Patterns of childhood trauma and psychological distress among injecting heroin users in China. PLoS ONE, 5 (12), e15882.

Widom, C. S., \& Kuhns, J. B. (1996). Childhood victimization and subsequent risk for promiscuity, prostitution, and teenage pregnancy: a prospective study. American Journal of Public Health, 86 (11), 1607-1612.

ABSTRACT - The study aims to investigate the prevalence of and factors associated with child poly-victimization, in order to provide a reliable estimate of the problem in the Chinese context. Using a 2-stage stratified sampling procedure, 18,341 high schools students were recruited from 6 cities in China. Students were asked to complete a self-administered questionnaire containing items about child victimization, addictive behaviors and adolescent sexual behaviors. In particular, the validated Chinese version of Juvenile Victimization Questionnaire (JVQ) was employed in this study. Precedingyear prevalence rates were $23.2 \%$ for any type of child victimization and $9.2 \%$ for 
poly-victimization. Living in Mainland China, being younger in age, and having siblings were associated with increased odds ratios of poly-victimization. Some adolescent addictive and sexual behaviors, including gambling, smoking, alcohol abuse, early sexual intercourse, multiple sex partners and teenage pregnancy, were also significantly associated with increased likelihood of poly-victimization. The findings of this study provide evidence of strong associations between child victimization and risky behaviors, which warrant prioritized intervention in cases of poly-victimization.

KEYWORDS - Child victimization, addictive behavior, sexual behavior, poly-victimization, China.

RESUMEN - Este estudio apunta a evaluar la prevalencia y los factores asociados a la poli-victimización del niño así como también a proporcionar una estimación fiable del problema en el contexto chino. Utilizando un procedimiento de muestreo estratificado en dos niveles, 18.341 alumnos chinos han sido reclutados en seis ciudades de China. Los mismos han sido invitados a completar un cuestionario auto-administrado que contenía preguntas sobre sus victimizaciones en la infancia, sus comportamientos adictivos y sobre los comportamientos sexuales en la adolescencia. La versión china validada del cuestionario sobre la victimización juvenil (JVQ por el acrónimo anglófono) ha sido utilizada. Los índices de prevalencia obtenidos en referencia al año precedente son de $23,2 \%$ por todo tipo de victimización de niños y 9,2\% por la poli-victimizacion. Vivir en China continental, estar entre los más jóvenes y tener hermanos son los factores asociados al aumento del riesgo ("odds ratio») de poli-victimización. Algunos comportamientos adictivos y sexuales de los adolescentes, incluyendo los juegos de azar, el tabaquismo, el alcoholismo, las relaciones sexuales precoces, las parejas sexuales múltiples, y el embarazo adolescente, están asociados significativamente a un aumento de la probabilidad de poli-victimización. Los resultados del presente estudio revelan fuertes asociaciones entre la victimización de los niños y las conductas de riesgo, subrayando así la necesidad de una intervención prioritaria en el caso de una poli-victimización.

PALABRAS CLAVE • Victimización de niños, comportamientos adictivos, comportamiento sexual, poli-victimización, China. 